\title{
Influence of Self-excited Vibrating Cavity Structure on Droplet Diameter Characteristics of Twin-fluid Nozzle
}

Bo Chen', Dian-Rong Gao ${ }^{1 *}$, Shao-Feng Wu² and Jian-Hua Zhao ${ }^{1,3}$

\begin{abstract}
It is a great challenge to find effective atomizing technology for reducing industrial pollution; the twin-fluid atomizing nozzle has drawn great attention in this field recently. Current studies on twin-fluid nozzles mainly focus on droplet breakup and single droplet characteristics. Research relating to the influences of structural parameters on the droplet diameter characteristics in the flow field is scarcely available. In this paper, the influence of a self-excited vibrating cavity structure on droplet diameter characteristics was investigated. Twin-fluid atomizing tests were performed by a self-built open atomizing test bench, which was based on a phase Doppler particle analyzer (PDPA). The atomizing flow field of the twin-fluid nozzle with a self-excited vibrating cavity and its absence were tested and analyzed. Then the atomizing flow field of the twin-fluid nozzle with different self-excited vibrating cavity structures was investigated. The experimental results show that the structural parameters of the self-excited vibrating cavity had a great effect on the breakup of large droplets. The Sauter mean diameter (SMD) increased with the increase of orifice diameter or orifice depth. Moreover, a smaller orifice diameter or orifice depth was beneficial to enhancing the turbulence around the outlet of nozzle and decreasing the SMD. The atomizing performance was better when the orifice diameter was $2.0 \mathrm{~mm}$ or the orifice depth was $1.5 \mathrm{~mm}$. Furthermore, the SMD increased first and then decreased with the increase of the distance between the nozzle outlet and self-excited vibrating cavity, and the SMD of more than half the atomizing flow field was under $35 \mu \mathrm{m}$ when the distance was $5.0 \mathrm{~mm}$. In addition, with the increase of axial and radial distance from the nozzle outlet, the SMD and arithmetic mean diameter (AMD) tend to increase. The research results provide some design parameters for the twin-fluid nozzle, and the experimental results could serve as a beneficial supplement to the twin-fluid nozzle study.
\end{abstract}

Keywords: Atomizing nozzle, Twin-fluid, Sauter mean diameter, Arithmetic mean diameter, Self-excited vibrating cavity, Phase Doppler particle analyzer

\section{Introduction}

With the increasing consumption of fossil fuels and the heavy emissions of dust from industry, serious environmental and health problems have drawn great attention from the public in recent years [1,2]. Micron and submicron droplets have made significant contributions to reducing exposure to fine particles. Atomization and cavitation are the two important approaches to generating

\footnotetext{
*Correspondence: gaodr@ysu.edu.cn

${ }^{1}$ School of Mechanical Engineering, Yanshan University,

Qinhuangdao 066004, China

Full list of author information is available at the end of the article
}

droplets [3-5]. Owing to the smaller droplets size, larger coverage of droplets, and wider applicability, the excellent performance of atomizing technology has motivated intensive studies in many fields [6-9]. However, the spray process of a twin-fluid nozzle involves multiphase coupling, dynamic changing, evaporation, and condensation, which have great influence on the stability and uniformity of droplets in the spray field and the atomizing efficiency of the twin-fluid nozzle. Therefore, it would be a great accomplishment if the fine droplet mists could be generated steadily through changing the structure of twin-fluid nozzle. 
Many efforts have been made by scholars around the world to improve the atomizing performance of spray nozzles in the past decades, such as designing nozzle structures $[10,11]$, innovating atomizing methods [12, $13]$, and optimizing operating conditions [14, 15]. Presently, it is believed that fine and uniform droplet mists can be obtained easily by the twin-fluid nozzle atomizing technology combined with external forces, and this was demonstrated to be a promising method $[12,16]$.

Electrostatically charged and acoustic waves vibration are the two main incentive approaches, which provide a new mentality for the improvement of the nozzle atomizing performance. In fact, as early as 1984, Law et al. [17] proposed the measuring of charged droplets by laser Doppler anemometry. Furthermore, they studied the trajectories of droplets in an electric field, which has profound consequences for the electrostatic crop spraying of foliar tips. Wang et al. $[16,18]$ analyzed the charged twin-fluid spray structure, the droplet diameter, the axial jet velocity, and the turbulent fluctuation intensity in an electrostatic charged spray by particle image velocimetry (PIV) and a phase Doppler particle analyzer (PDPA). Shi et al. [19] investigated the spray angles under a stable cone-jet mode in an electro-spraying experiment under single and combined electric fields. Moreover, they declared that both electrode parameters and space charges have a significant impact on the electric field distribution. However, safety of the charged droplets and electrostatically charged process are difficult to ensure in some places, such as coal mines, high concentration dust areas.

Based on the acoustic waves vibration technology, Grant [20] applied ultrasonic gas atomization to an injection spray. Narayanan et al. [21, 22], ascertained the effects of the vibrating cavity structure on frequency and amplitude characteristics through contrasting experiments. In addition, $\mathrm{Zu}$ et al. [23] researched the characteristics of air vibration in a Hartmann resonance cavity with/without actuators. The result indicated that actuators enhance the strength and onset process of oscillating remarkably. Ruan et al. [24] investigated the effects of geometric parameters on the flow and acoustic field characteristics of an ultrasonic atomizer by experimentation and numerical simulation. However, most of these studies mainly focused on the characteristics of acoustics and air flow fields, but have not taken liquid into consideration.

Rajan et al. [25] pointed out that finer droplets with high sphericity and uniform size distribution could be achieved by ultrasonic energy. Compared to the conventional nozzles, the atomizing process induced by ultrasound had a lower energy consumption. Narayanan et al. [26] investigated the effects of Hartmann cavity acoustics on the droplets atomization by experimentation. The results revealed that the existence of a sound field led to the droplets undergoing large deformation and becoming irregular in shape. Ferreira et al. [27] experimentally investigated the influence of different structural parameters and flow conditions on the droplets size of a twin-fluid nozzle with an internal mixing chamber. Meanwhile, the main parameters of optimum results had been determined. Li et al. [28] researched the effects of jetting distance and impacts of the collision wall angle on the outlet velocity based on the computational fluid dynamics (CFD) numerical simulation technology. Liu et al. [29] studied the influence of air pressure on droplet diameter, atomization angle and water consumption of a modified ultrasonic nozzle. According to the achievements of former researchers, it can be considered that fine droplet mists and uniform droplet size distribution can be achieved in an acoustic field, which could be generated by a vibrating cavity structure of the twin-fluid nozzle. Acoustic field characteristics of twin-fluid nozzles have been mainly investigated in recent years. As we know, droplet diameter characteristics are the important indexes of twin-fluid atomizing performance, but investigation of the influence of vibrating cavity structure on that is reported rarely.

Exploring the influence of a vibrating cavity structure on droplet diameter characteristics is beneficial for recognizing the atomizing principles, which is a critical factor to improve the atomizing performance of the twinfluid nozzle. On the basis of previous studies [29-31], by using an open atomizing test bench which is based on a PDPA, this study is mainly focused on the influence of a self-excited vibrating cavity structure on the droplet diameter characteristics of a twin-fluid nozzle.

\section{Experimental Details}

\subsection{Experimental Set-up and Measurement Technique}

The twin-fluid atomizing flow field was tested by a selfbuilt open atomizing test bench which was based on a PDPA. The working mediums of the twin-fluid atomizing tests were compressed air and clean water. A schematic diagram of the atomizing test bench is described in Figure 1. The air compressor (FB-0.36, XMEA) was connected with air pipelines which supplied compressed air for the twin-fluid nozzle. The water pump (XKJ$804 \mathrm{~S}, \mathrm{ZJLO}$ ) was connected with water pipelines which provided the high pressure water required. The system pressure was regulated by an air pressure reducing valve (TW3000, STNC) and a water pressure reducing valve (ML5201, ZJML). The values of flow rate and pressure in the pipelines could be recorded by the flowmeter (YK-LLQ, DLYK) and pressure gauge (SHFY Ltd., Y60), respectively. The air compressor and water pump might 


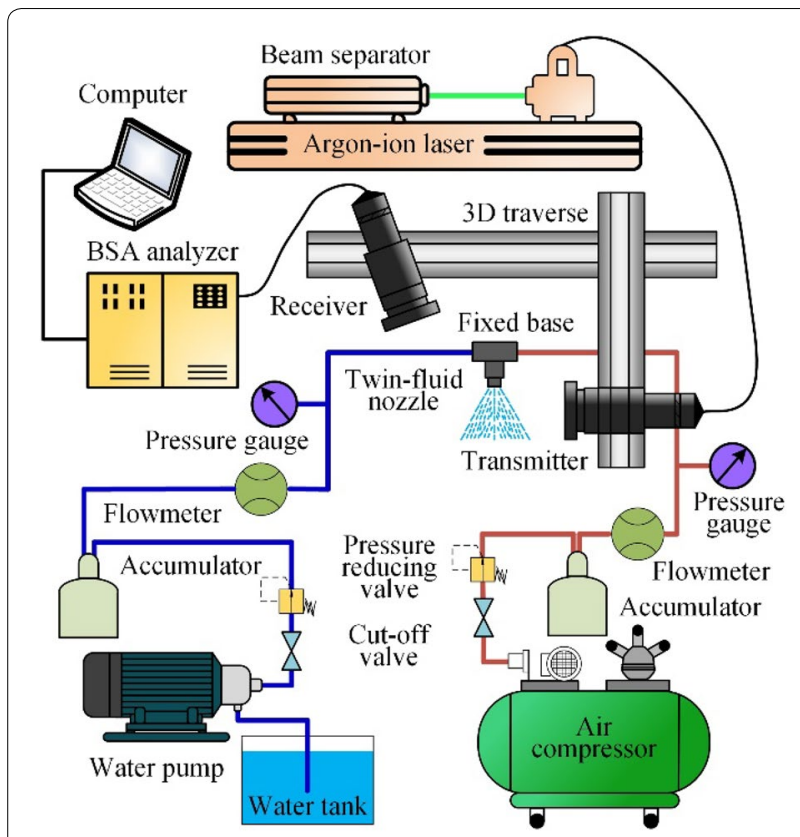

Figure 1 Schematic diagram of atomizing test bench

have caused the fluctuation of the working mediums in the pipelines when they worked, and the effect of fluctuation could be weakened by an accumulator (HGXQ, TJHLM). The twin-fluid nozzle was mounted to the fixed base, which was also connected with air pipelines and water pipelines on both sides, so that the compressed air and water could flow into the nozzle.

A phase Doppler particle analyzer (Dantec) was used for droplet diameter measurement. The PDPA performed real-time measurements with high precision $(0.5 \%)$ and stability, and the measuring range of diameters was $0.5-13000 \mu \mathrm{m}$. The PDPA was comprised of an Argonion laser, a beam separator, a transmitter, a receiver, an analyzer, and a computer. The laser was produced by the Argon-ion laser and transferred from beam separator to transmitter. The data collection focal point was generated by the laser from the transmitter, and the receiver collected the real-time signals and transmitted them to the BSA analyzer for data analysis. Results could be displayed through the computer and the location of the focal point would be adjusted by the 3D traverse.

\subsection{Test Nozzle Specification}

In order to investigate the influence of the self-excited vibrating cavity structure on the droplet diameter characteristics of the twin-fluid nozzle, specimens of twinfluid nozzles without a self-excited vibrating cavity and twin-fluid nozzles with different structures of self-excited vibrating cavities were prepared. A schematic diagram of the twin-fluid nozzle is described in Figure 2. The

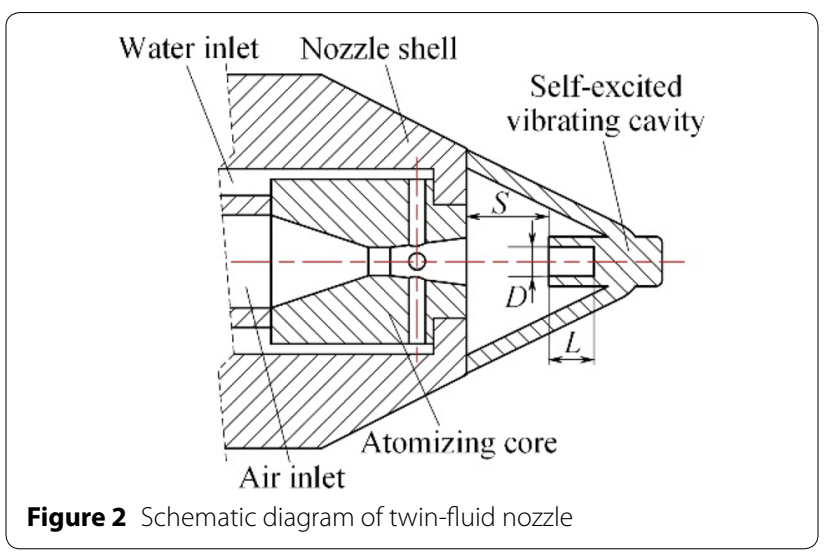

twin-fluid nozzle was mainly comprised of a self-excited vibrating cavity, atomizing core, and nozzle shell. Three different self-excited vibrating cavity structural parameters were mainly considered, which included the orifice diameter $(D)$ and orifice depth $(L)$ of the self-excited vibrating cavity, and the distance between the nozzle outlet and self-excited vibrating cavity $(S)$. Compressed air and water flowed into the atomizing core through the air inlet and water inlet, respectively. The first atomizing process occurred in the atomizing core. A large number of droplets erupted from the atomizing core and impacted the self-excited vibrating cavity strongly, which resulted in the secondary atomizing process occurring, so that the atomizing performance would be improved.

\subsection{Testing and Analytic Methods}

All twin-fluid atomizing tests were conducted in a closed environment. In order to keep the testing environment steady, the temperature of the environment was maintained at $24 \pm 1{ }^{\circ} \mathrm{C}$, the relative humidity was maintained between $45 \%-50 \%$, and the atmospheric pressure was about $102.6 \mathrm{kPa}$. In addition, the viscosity and surface tension of the water were $0.9142 \mathrm{mPa} \cdot \mathrm{s}$ and $0.0721 \mathrm{~N} / \mathrm{m}$, respectively.

The air compressor and water pump were opened at the beginning of test, and then the operating parameters adjusted as required. For ensuring that the atomizing flow field was steady, the data collecting system would be started five seconds after the nozzle began to spray. The time of testing was sustained for $30 \mathrm{~s}$, then the data would be recorded and processed. In order to ensure the accuracy and repeatability of results, all the recorded data were the average of three replicates.

3-D measurements would be replaced by a planar method for improving the efficiency of the flow field measurements. The schematic of planar measurement points is shown in Figure 3. Based on the results of these measurement points, 2-D contour plots were 


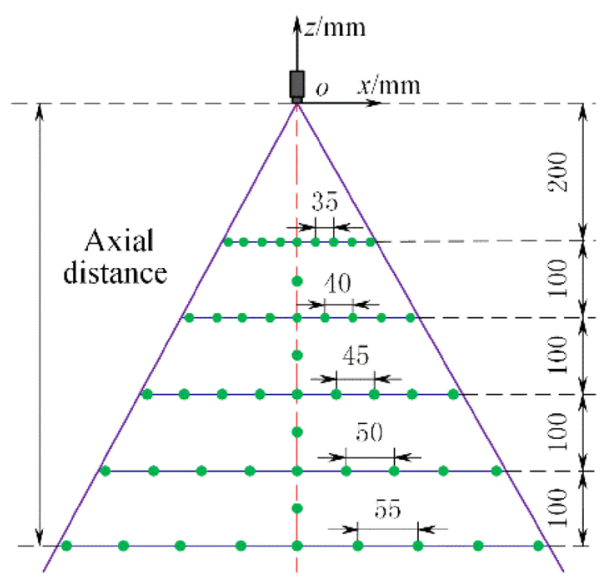

Figure 3 Schematic of planar measurement points

adopted to describe the spatial distribution of droplet diameter characteristics more clearly [32]. The droplet diameter characteristics of the overall atomizing process could be analyzed in detail. All the tests at different measurement points were conducted under the same operating conditions. The air flow rate was $1.0 \mathrm{~m}^{3} / \mathrm{h}$ and the water flow rate was $30 \mathrm{~L} / \mathrm{h}$. Meanwhile, the droplet diameters analyzed included the Sauter mean diameter (SMD) and Arithmetic mean diameter (AMD). The definition of SMD and AMD are as follows:

$$
\begin{aligned}
& S M D=\sum_{i} N_{i} D_{i}^{3} / \sum_{i} N_{i} D_{i}^{2}, \\
& A M D=\sum_{i} N_{i} D_{i}^{1} / \sum_{i} N_{i} D_{i}^{0},
\end{aligned}
$$

where $N_{i}$ is the number of droplet, and $D_{i}$ is the diameter of droplet $i$.

\section{Results and Discussion}

\subsection{Influence of the Self-excited Vibrating Cavity and Its Absence on Droplet Diameter Characteristics}

\subsubsection{SMD Spatial Distribution Analysis}

Figure 4 presents the SMD spatial distribution of the twin-fluid nozzle with a self-excited vibrating cavity ( $D=2.0 \mathrm{~mm}, L=1.5 \mathrm{~mm}, S=4.0 \mathrm{~mm}$ ) and its absence. As seen, the two twin-fluid nozzles have a similar rule that the SMD was smaller when closer to the nozzle outlet. The SMD increased along the axial direction (correspond to the $-z$ in Figure 3 ) and radial direction (correspond to the $\pm x$ in Figure 3 ) of nozzle. It demonstrated the same regularity on both sides of the axial line, and the SMD was larger on the boundaries. It is known that the turbulence around the outlet of nozzle is the most intense [30], so the breakup of droplets is enhanced which results in the SMD decrease. Because of heat and mass transfer, collision coalescence, and condensation, the SMD will increase with the movement of droplets. In addition, it is easier for large droplets to move outward than fine droplets due to the larger momentum, which could also cause the increase of SMD at the boundary.

Compared to the SMD spatial distribution of the twin-fluid atomizing nozzle without a self-excited vibrating cavity, it can be concluded that the SMD was smaller and the coverage of droplets was bigger for the nozzle with a self-excited vibrating cavity. The strong collision of droplets between the nozzle outlet and vibrating cavity lead to the increase of the droplets' radial velocity, which caused the coverage of droplets to increase remarkably. As seen, the SMD near the nozzle

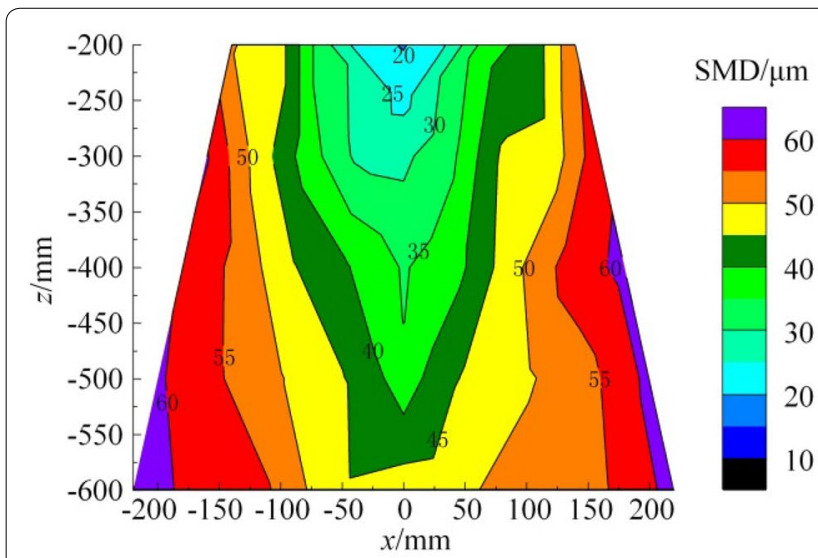

a With self-excited vibrating cavity

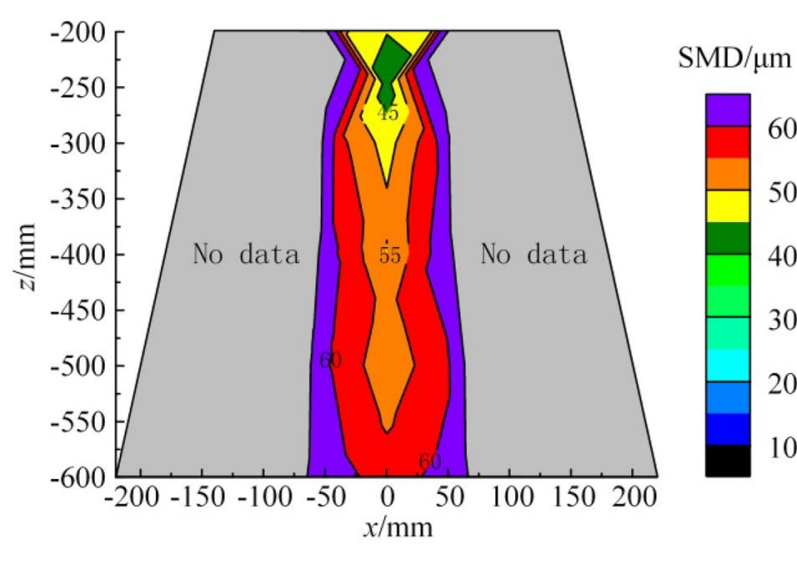

b Without self-excited vibrating cavity

Figure 4 SMD spatial distribution of twin-fluid nozzle with self-excited vibrating cavity and its absence 
outlet without a self-excited vibrating cavity was about two times larger than that with a self-excited vibrating cavity. This result is due to the acoustic field which was generated by the intense oscillations of the self-excited vibrating cavity. Furthermore, this phenomenon can prove that the acoustics are beneficial to enhancing the atomization. Narayanan [26] had a similar discovery in the research of atomization in the acoustic field of a Hartmann whistle.

\subsubsection{Comparative Analysis of SMD and AMD}

The SMD and AMD in measurement points $400 \mathrm{~mm}$ away from the nozzle along the axial direction are shown in Figure 5. As seen, with the increase of radial distance, the SMD increased remarkably, and a similar principle is shown in the AMD as well. Moreover, it is clear that the SMD and AMD were smaller for the nozzle with the self-excited vibrating cavity; this phenomenon can also ensure that the self-excited vibrating cavity had a great effect on decreasing the droplet diameter. In addition, there are obvious differences between the SMD and AMD in both of the two nozzles, which are mainly due to the wide range of the droplet diameter distribution. It is known that the SMD will increase obviously with the number of large droplets growing, but that the AMD is not influenced seriously [33]. The difference in SMD is bigger than in AMD of the two nozzles, which reveals that it was beneficial for the breakup of large droplets when the nozzle with a self-excited vibrating cavity was used.

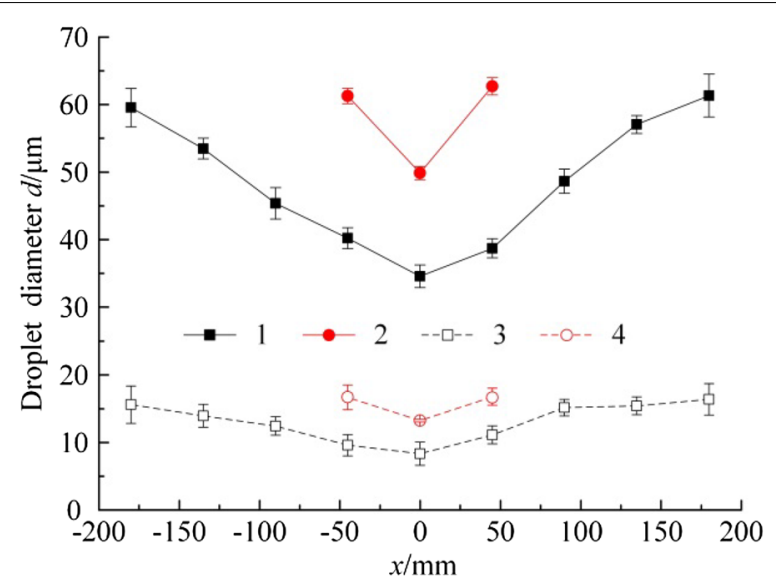

Figure 5 Comparative analysis of SMD and AMD. 1. SMD of twin-fluid nozzle with self-excited vibrating cavity; 2 . SMD of twin-fluid nozzle without self-excited vibrating cavity; 3 . AMD of twin-fluid nozzle with self-excited vibrating cavity; 4. AMD of twin-fluid nozzle without self-excited vibrating cavity

\subsection{Influence of the Orifice Diameter on Droplet Diameter Characteristics}

\subsubsection{SMD Spatial Distribution Analysis}

Figure 6 presents the SMD spatial distribution of twinfluid nozzles with different orifice diameters of the self-excited vibrating cavity $(L=1.5 \mathrm{~mm}, S=4.0 \mathrm{~mm})$. As seen, the SMD spatial distributions of different orifice diameters showed the same regularity. The SMD increased with the increase of axial and radial distance from the nozzle outlet, and the SMD near the nozzle outlet was smaller than that in other zones.

It is clear that the SMD of droplets increased with the increase of the orifice diameter of the self-excited vibrating cavity. The atomizing performance was superior when the orifice diameters were $1.0 \mathrm{~mm}$ and $1.5 \mathrm{~mm}$, and the SMD of more than half the atomizing flow field was under $35 \mu \mathrm{m}$. With the orifice diameter continuing to increase to $2.5 \mathrm{~mm}$, the SMD increased slowly and the atomizing performance became worse. This is due to the decrease of acoustic field frequency, because Ruan et al. [24] reported that acoustic field frequency showed a decreasing trend with the increase of the orifice diameter of the vibrating cavity. Furthermore, the high acoustic field frequency had a great effect on improving the turbulence of the fluid field which was beneficial for decreasing the droplet diameter. The results reveal that a better SMD spatial distribution was obtained when the self-excited vibrating cavity had a smaller orifice diameter.

\subsubsection{Comparative Analysis of SMD and AMD}

The SMD and AMD of different orifice diameters in measurement points $400 \mathrm{~mm}$ away from the nozzle along the axial direction are shown in Figure 7. The SMD and AMD show increasing trends with the increase of radial distance from the central axis of the atomizing flow field. With the increase of orifice diameter, the SMD and AMD show an increasing trend as well, but the trend of AMD is obviously less than that of SMD. According to the above analysis, it shows that the SMD can reflect the changes of large droplet diameters, while the AMD is not sensitive to the changes of large droplet diameters. It indicates that the decrease of the orifice diameter of the self-excited vibrating cavity was beneficial for the breakup of large droplets, which mainly owed to the increase of acoustic field frequency.

\subsection{Influence of the Orifice Depth on Droplet Diameter Characteristics}

\subsubsection{SMD Spatial Distribution Analysis}

The SMD spatial distribution of the twin-fluid nozzle with different orifice depths of the self-excited vibrating cavity $(D=2.0 \mathrm{~mm}, S=4.0 \mathrm{~mm})$ is shown in 

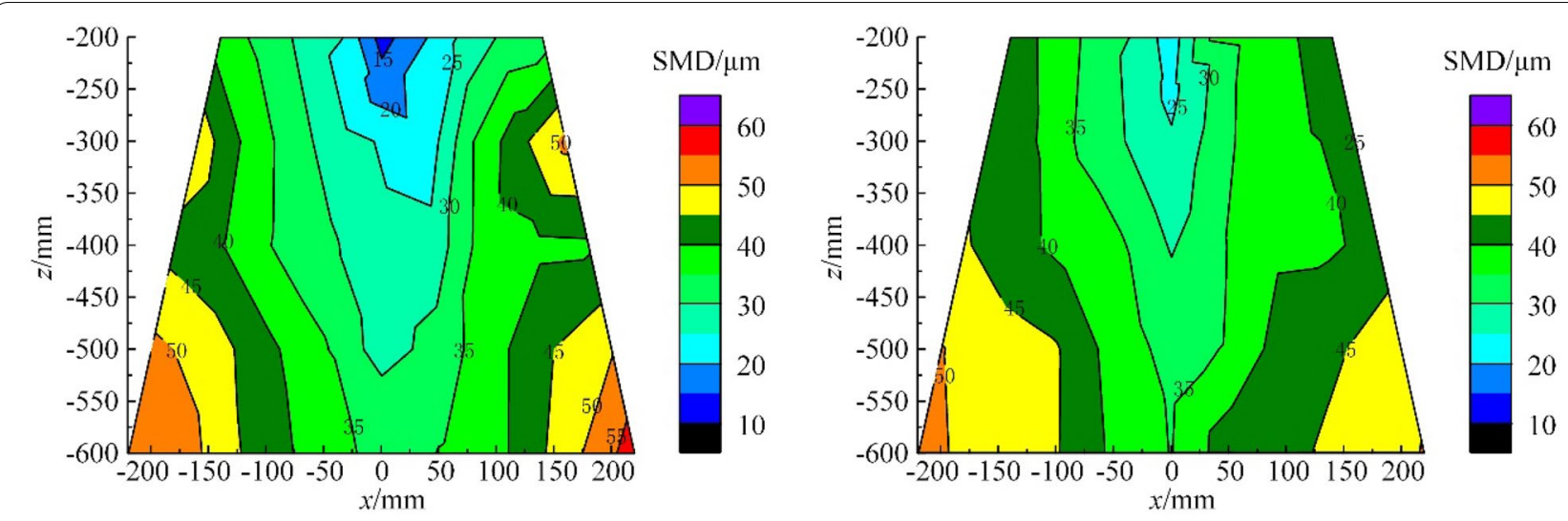

a $D=1.0 \mathrm{~mm}$

b $D=1.5 \mathrm{~mm}$
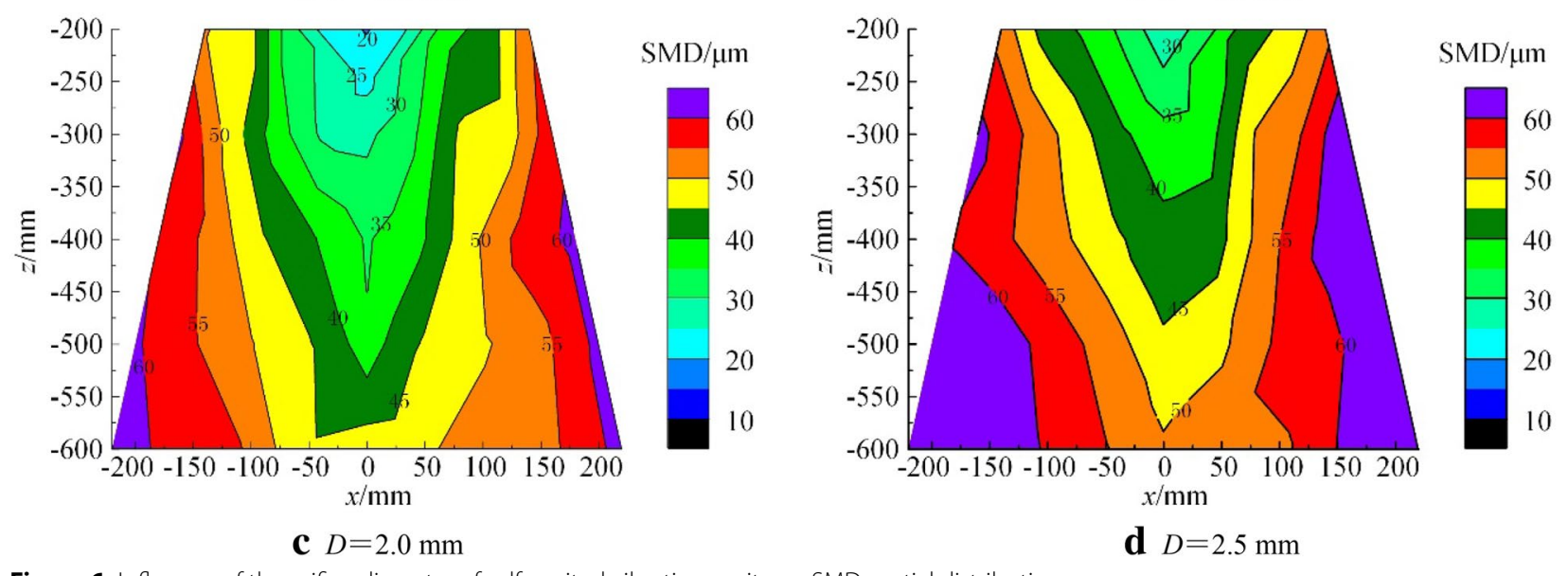

Figure 6 Influence of the orifice diameter of self-excited vibrating cavity on SMD spatial distribution

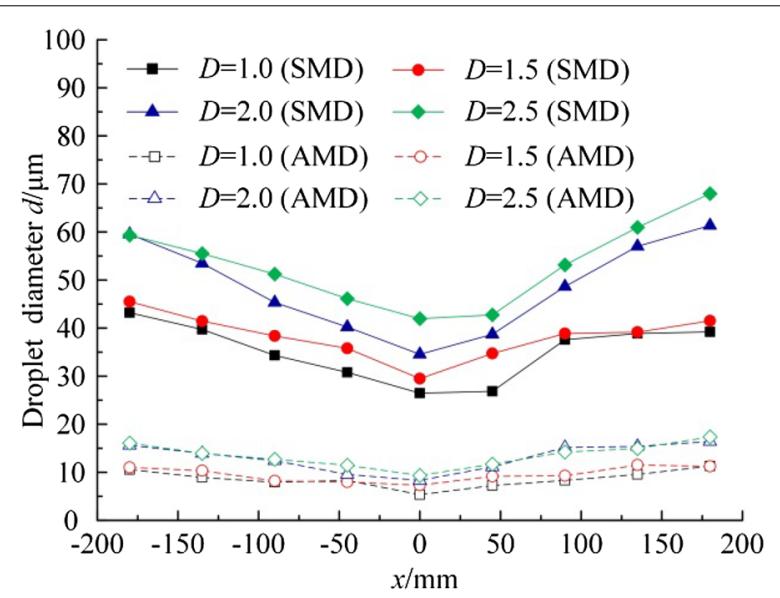

Figure $7 \mathrm{SMD}$ and $\mathrm{AMD}$ analysis of different orifice diameters

Figure 8 . The SMD of droplets were gradually changed from small to large as the droplets moved away from the nozzle outlet, and the SMD along the radial direction grew faster than that along the axial direction. The main reason for that is the increase of droplets' radial velocity after collision. Due to the high acoustic field frequency, the turbulence and collision in the region between the nozzle outlet and self-excited vibrating cavity were quite intensive, which lead to the increase of the droplets' radial velocity.

As seen in Figure 8, the SMD of droplets show an increasing trend with the increase of the orifice depth of the self-excited vibrating cavity. The SMD of more than half the atomizing flow field was under $35 \mu \mathrm{m}$ when the orifice depth was $1.0 \mathrm{~mm}$, especially more fine droplets generated in the region around the nozzle outlet. However, with the orifice depth increase to $2.0 \mathrm{~mm}$, the atomizing performance turned poor, and the SMD increased in both the central and boundary area. This can be explained by the decrease of acoustic field frequency with the orifice depth increase, which resulted in a decrease of fluid field turbulence and an increase of droplet diameter. The empirical formula between acoustic field frequency and orifice depth can be defined as follows [34]: 

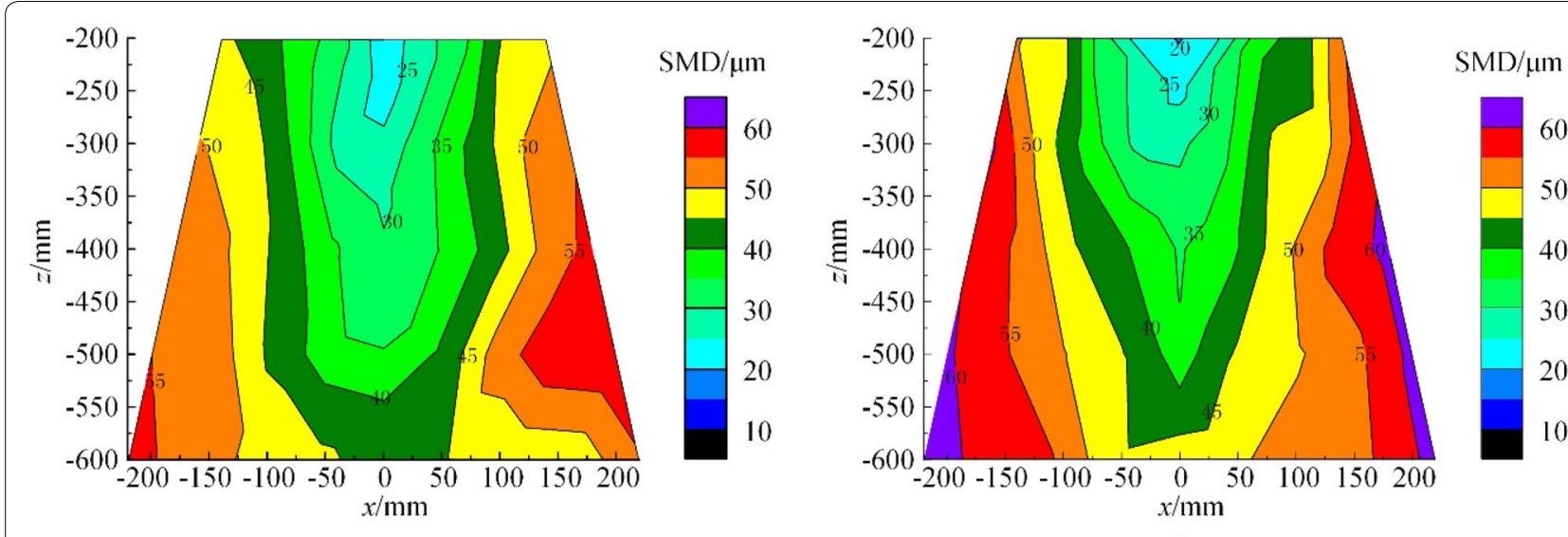

a $L=1.0 \mathrm{~mm}$

b $L=1.5 \mathrm{~mm}$

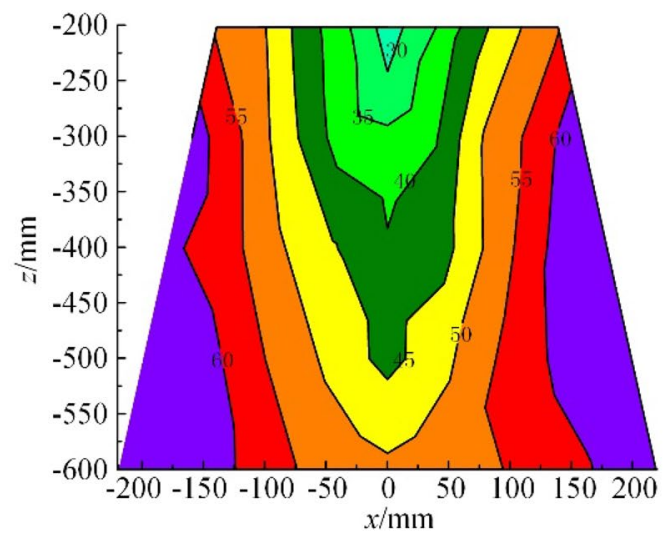

$\mathrm{SMD} / \mu \mathrm{m}$
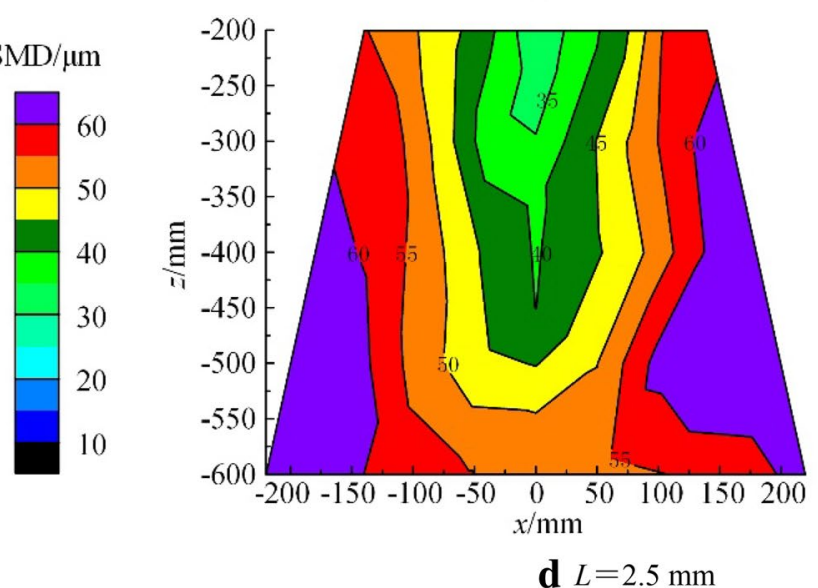

$\mathrm{SMD} / \mu \mathrm{m}$

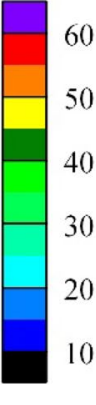

c $L=2.0 \mathrm{~mm}$

d $L=2.5 \mathrm{~mm}$

Figure 8 Influence of orifice depth of self-excited vibrating cavity on SMD spatial distribution

$$
f=\frac{c}{4(L+0.3 D)},
$$

where $f$ is the acoustic field frequency, $c$ is the acoustic velocity, $L$ is the orifice depth of the self-excited vibrating cavity, and $D$ is the orifice diameter of the self-excited vibrating cavity.

The SMD changed slowly when the orifice depth increased from $2.0 \mathrm{~mm}$ to $2.5 \mathrm{~mm}$, and the SMD spatial distribution was similar. Furthermore, the influence of the acoustic field frequency decrease on the SMD shows that a weakening trend can be indicated when the orifice depth was above $2.0 \mathrm{~mm}$. This result reveals that the SMD of droplets was finer when the orifice depth of the self-excited vibrating cavity was smaller.

\subsubsection{Comparative Analysis of SMD and AMD}

The SMD and AMD of different orifice depths in measurement points $400 \mathrm{~mm}$ away from the nozzle along the axial direction are shown in Figure 9. As seen, the SMD

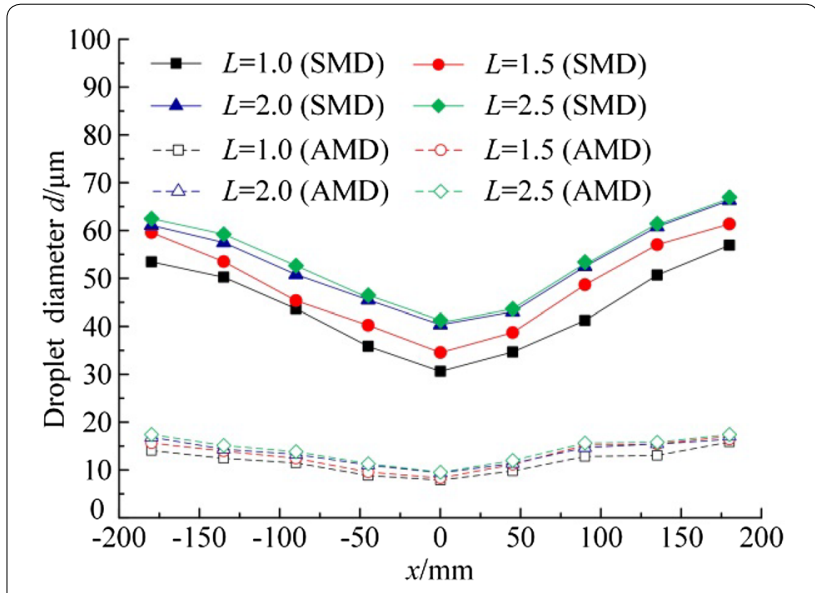

Figure $9 \mathrm{SMD}$ and $\mathrm{AMD}$ analysis of different orifice depth

and AMD increased with the increase of radial distance from the central axis of the atomizing flow field. With the increase of orifice depth, the SMD and AMD show 
increasing trends, but the change of AMD is still obviously less than SMD. The same regularity is displayed above and the reason is explained in Section 3.2.2. In addition, it confirms that the decrease of orifice depth of the self-excited vibrating cavity had a great effect on breaking up the large droplets into small droplets.

\subsection{Influence of the Distance between the Nozzle Outlet and Self-excited Vibrating Cavity on Droplet Diameter Characteristics}

\subsubsection{SMD Spatial Distribution Analysis}

The SMD spatial distribution of twin-fluid nozzles with different distances between the nozzle outlet and the self-excited vibrating cavity $(D=2.0 \mathrm{~mm}, L=1.5 \mathrm{~mm})$ are shown in Figure 10. As seen, the SMD of the droplets increases first and then decreases with the increase of $S$. Meanwhile, the four different distances have approximate SMD changing rates in the central and boundary zone. The obvious changes of SMD are displayed around the boundary zone of the four different distances. Moreover, a superior atomizing performance is obtained when the $S$ is $5.0 \mathrm{~mm}$, and the SMD of more than half the atomizing flow field is under $35 \mu \mathrm{m}$.

From the above analysis, it is known that the structural changes of the self-excited vibrating cavity have great influence on the droplet diameter by influencing the acoustic field frequency. Ruan and Narayanan [24, 26] had a similar discovery; that the acoustic field frequency showed a large oscillation, and the frequency decreased first and then increased with the increase of $S$. Therefore, a good, consistent relationship between the SMD decrease and the acoustic field frequency increase is presented.

\subsubsection{Comparative Analysis of SMD and AMD}

The SMD and AMD of different $S$ values in measurement points $400 \mathrm{~mm}$ away from the nozzle along the axial direction are shown in Figure 11. With the increase of radial distance from the central axis of the atomizing flow field, the SMD and AMD increase obviously. Meanwhile, the SMD and AMD show the same variation regularity in that they increase first and then decrease with
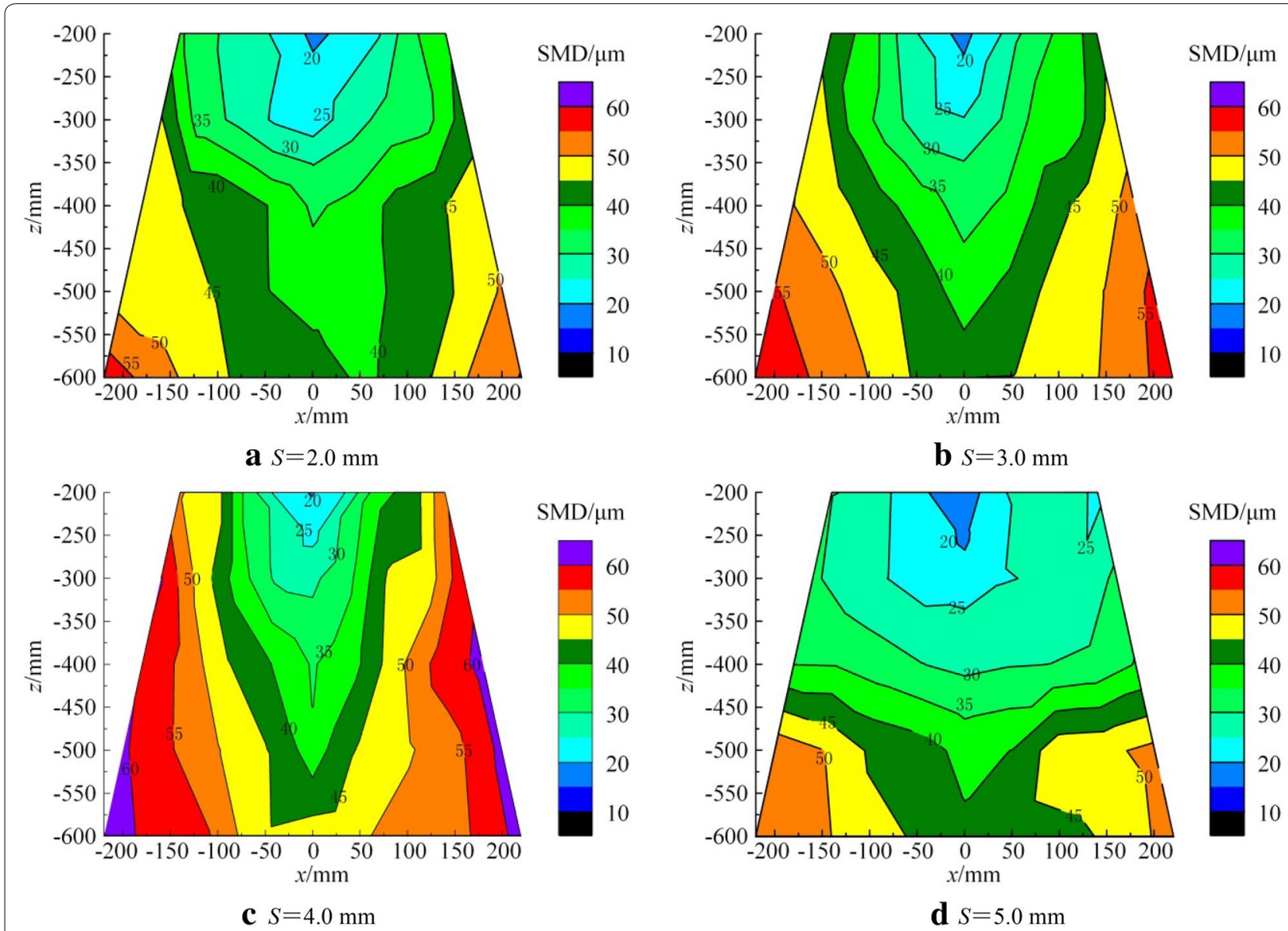

Figure 10 Influence of the distance between nozzle outlet and self-excited vibrating cavity on SMD spatial distribution 


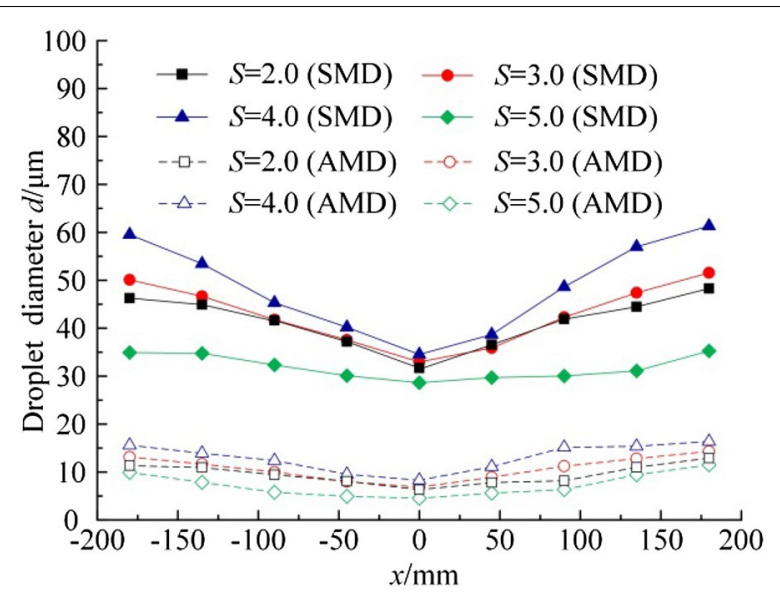

Figure 11 SMD and AMD analysis of different distances between nozzle outlet and self-excited vibrating cavity

the increase of $S$. In addition, the change of AMD is still obviously less than that of SMD in Figure 11. This phenomenon can prove once again that the acoustic field frequency produced by the twin-fluid nozzle has a great effect on the breakup of large droplets. Meanwhile, it can prove the correctness of the above analysis as well.

\section{Conclusions}

(1) A self-excited vibrating cavity was verified to be an effective key for improving the atomizing performance of a twin-fluid nozzle. The structural parameters of the self-excited vibrating cavity had a great effect on the breakup of large droplets.

(2) The SMD increased with the increase of orifice diameter or orifice depth of the self-excited vibrating cavity. A good, consistent relationship between the SMD decrease and the acoustic field frequency increase was presented. A smaller orifice diameter or orifice depth is beneficial to enhancing the turbulence around the outlet of nozzle and decreasing the SMD. The atomizing performance was better when the orifice diameter was $2.0 \mathrm{~mm}$ or the orifice depth was $1.5 \mathrm{~mm}$.

(3) SMD increased first and then decreased with the increase of the distance between the nozzle outlet and the self-excited vibrating cavity. A superior atomizing performance was obtained when the distance was $5.0 \mathrm{~mm}$, and the SMD of more than half the atomizing flow field was under $35 \mu \mathrm{m}$.

(4) With axial and radial distance increase, the SMD and AMD tended to increase. Meanwhile, the SMD and AMD near the nozzle outlet zone were smaller than those in other zones. High acoustic field fre- quency could exacerbate the breakup of droplets, and the distribution of droplet diameter become wider, which caused an obvious difference between the SMD and AMD.

\section{Authors' Contributions}

D-RG was in charge of the whole trial; BC wrote the manuscript; S-FW and $\mathrm{J}-\mathrm{HZ}$ assisted with sampling and laboratory analyses. All authors read and approved the final manuscript.

\section{Author details}

${ }^{1}$ School of Mechanical Engineering, Yanshan University, Qinhuangdao 066004, China. ${ }^{2}$ School of Mechanical Engineering, Hangzhou Dianzi University, Hangzhou 310018, China. ${ }^{3}$ State Key Laboratory of Fluid Power and Mechatronic Systems, Zhejiang University, Hangzhou 310027, China.

\section{Authors' Information}

Bo Chen, born in 1990, is currently a PhD candidate at School of Mechanical Engineering, Yanshan University, China. His main research interests include fluid machinery and multiphase flow.

Diao-Rong Gao, born in 1962, is currently a professor and a PhD candidate supervisor at School of Mechanical Engineering, Yanshan University, China. He received his PhD degree from Yanshan University, China, in 2001. His research interests include CFD, PIV, heavy machinery fluid transmission and control and new types of fluid components and devices.

Shao-Feng Wu, born in 1987, is currently a lecturer at School of Mechanical Engineering, Hangzhou Dianzi University, China. He received his PhD degree from Yanshan University, China, in 2017. His research interests include fluid transmission and control and fluid machinery.

Jian-Hua Zhao, born in 1983, is currently an associate professor at School of Mechanical Engineering, Yanshan University, China. He received his PhD degree from Yanshan University, China, in 2013. His research interests include the simulation and analysis of hydrostatic bearing.

\section{Competing Interests}

The authors declare that they have no competing interests.

\section{Funding}

Supported by National Natural Science Foundation of China (Grant No. 51705445), Hebei Provincial Natural Science Foundation of China, (Grant No. E2016203324), and Open Foundation of the State Key Laboratory of Fluid Power and Mechatronic Systems of China (Grant No. GZKF-201714).

\section{Publisher's Note}

Springer Nature remains neutral with regard to jurisdictional claims in published maps and institutional affiliations.

Received: 16 June 2017 Accepted: 14 August 2018

Published online: 23 August 2018

\section{References}

[1] A Fuertes, M Casals, M Gangolells, et al. An environmental impact causal model for improving the environmental performance of construction processes. Journal of Cleaner Production, 2013, 52: 425-437.

[2] E R Kohlman-Rabbani, A Shapira, A R B Martins, et al. Characterization and evaluation of dust on building construction sites in brazil. Neurochemistry International, 2014, 5(1): 1-8.

[3] M Zaremba, L Weiß, M Malý, et al. Low-pressure twin-fluid atomization: effect of mixing process on spray formation. International Journal of Multiphase Flow, 2017, 89: 277-289.

[4] J Zou, Y Zhu, M Pan, et al. A study on cavitation erosion behavior of $\mathrm{AlSi}_{10}$ Mg fabricated by selective laser melting (SLM). Wear, 2017, 376: 496-506.

[5] Y Zhu, J Zou, W L Zhao, et al. A study on surface topography in cavitation erosion tests of $\mathrm{AlSi}_{10} \mathrm{Mg}$. Tribology International, 2016, 102: 419-428. 
[6] F C Lujaji, A A Boateng, M Schaffer, et al. Spray atomization of bio-oil/ ethanol blends with externally mixed nozzles. Experimental Thermal and Fluid Science, 2016, 71: 146-153.

[7] A A Shraiber, V V Dubrovsky, A M Podvysotsky. Experimental study of the laws of interaction between small particles and large drops. Atomization and Sprays, 2014, 24(11): 937-947.

[8] S G Daviault, O B Ramadan, E A Matida, et al. Atomization performance of petroleum coke and coal water slurries from a twin fluid atomizer. Fuel, 2012, 98: 183-193.

[9] J Park, J Kang, S Park. Comparisons of spray characteristics depending on the nozzle parameters in a wafer cleaning system using a twin-fluid nozzle. Journal of the Electrochemical Society, 2016, 163(3): 88-96.

[10] A Sander, T Penović. Droplet size distribution obtained by atomization with two-fluid nozzles in a spray dryer. Chemical Engineering and Technology, 2014, 37(12): 2073-2084.

[11] J Karnawat, A Kushari. Controlled atomization using a twin-fluid swirl atomizer. Experiments in Fluids, 2006, 41 (4): 649-663.

[12] M Dobre, L Bolle. Practical design of ultrasonic spray devices: experimental testing of several atomizer geometries. Experimental Thermal and Fluid Science, 2002, 26(2): 205-211.

[13] A Rezvanpour, EW C Lim, C H Wang. Computational and experimental studies of electrohydrodynamic atomization for pharmaceutical particle fabrication. AlChE Journal, 2012, 58(11): 3329-3340.

[14] D Nuyttens, K Baetens, M D Schampheleire, et al. Effect of nozzle type, size and pressure on spray droplet characteristics. Biosystems Engineering, 2007, 97(3): 333-345.

[15] J F Wang, S C Zhang, Z W Zuo. Experimental study of influence rules on spray charged characteristics in the induction charging process. High Voltage Engineering, 2017, 43(2): 514-519. (in Chinese)

[16] ZT Wang, Q G Cen, X N Song, et al. Experiment of twin-fluid charged spray by PDA. Transactions of the Chinese Society of Agricultural Machinery, 2008, 39(10): 80-84. (in Chinese)

[17] S E Law, A G Bailey. Perturbations of charged-droplet trajectories caused by induced target corona: LDA analysis. IEEE Transactions on Industry Applications, 1984, 20(6): 1613-1622.

[18] J F Wang, ZTWang, H M MAO, et al. Measurement of twin-fluid electrostatic spray structure by using PIV. Transactions of the Chinese Society for Agricultural Machinery, 2009, 40(9): 107-111. (in Chinese)

[19] Y L Shi, Z B Luo, Y H Gan, et al. Analysis on electric field strength distribution in spraying region of small-scale electro-spraying system. Transactions of the Chinese Society for Agricultural Machinery, 2016, 47(5): 343-351. (in Chinese)
[20] N J Grant. Rapid solidification of metallic particulates. JOM, 1983, 35(1): 20-27.

[21] S Narayanan, K Srinivasan, T Sundararajan. Acoustic characteristics of external chamfered Hartmann whistles. Applied Acoustics, 2013, 74(9): 1104-1116.

[22] S Narayanan, K Srinivasan, T Sundararajan. Aero-acoustic features of internal and external chamfered Hartmann whistles: A comparative study. Journal of Sound and Vibration, 2014, 333(3): 774-787.

[23] H B Zu, Z W Zhou, Z L Wang. Properties of acoustic resonance in doubleactuator ultra-sonic gas nozzle: numerical study. Applied Mathematics and Mechanics, 2012, 33(12): 1481-1492.

[24] C Ruan, Y Huang, J Q Cai, et al. Aeroacoustic and flow field features of ultrasonic atomizer based on Hartmann resonance tube. Journal of Aerospace Power, 2016, 31(9): 2104-2114. (in Chinese)

[25] R Rajan, A B Pandit. Correlations to predict droplet size in ultrasonic atomisation. Ultrasonics, 2001, 39(4): 235-255.

[26] S Narayanan, K Srinivasan, T Sundararajan. Atomization in the acoustic field of a Hartmann whistle. International Journal of Spray and Combustion Dynamics, 2013, 5(1): 1-24.

[27] G Ferreira, J A García, F Barreras, et al. Design optimization of twin-fluid atomizers with an internal mixing chamber for heavy fuel oils. Fuel Processing Technology, 2009, 90(2): 270-278.

[28] HX Li, Q Z Liu, Y P Liu, et al. Numerical simulation on atomization characteristics of ultrasonic vibration nozzle based on CFD. Chinese Journal of Vacuum Science and Technology, 2017, 37(1): 113-117. (in Chinese)

[29] X Z Liu, G J Gao. Atomization characteristics of ultrasonic nozzle with Hartmann whistle structure. Chinese Journal of Vacuum Science and Technology, 2016, 36(3): 268-272. (in Chinese)

[30] B Chen, D R Gao, C Yang, et al. Atomizing characteristics of twin-fluid impact nozzle based on PDPA. Transactions of the Chinese Society for Agricultural Machinery, 2017, 48(4): 362-369. (in Chinese)

[31] B Chen, D R Gao, C Yang, et al. Multi-objective intelligent collaborative optimization of structure parameters for high-power remote sprayer. Journal of Mechanical Engineering, 2017, 53(6): 166-175. (in Chinese)

[32] S Lee, S Park. Spray atomization characteristics of a GDI injector equipped with a group-hole nozzle. Fuel, 2014, 137: 50-59.

[33] A Jankowski, A Sandel, J Seczyk, et al. Analysis of fuel spray preparation for internal combustion engines. Journal of KONES Internal Combustion Engines, 2002, 9(1): 323-332.

[34] XX Sun. Recent research advances of ultrasonic atomizer. Industrial Furnace, 2004, 26(1): 19-24. (in Chinese)

\section{Submit your manuscript to a SpringerOpen ${ }^{\circ}$ journal and benefit from:}

- Convenient online submission

- Rigorous peer review

- Open access: articles freely available online

- High visibility within the field

Retaining the copyright to your article

Submit your next manuscript at springeropen.com 